\title{
THE ANALYSIS OF AIR.
}

By W. J. ATKINSON BUTTERFIELD, M.A., F.I.C.

(Read at the Meeting, May 5, 1909.)

THe methods for the analysis of air which are about to be described have for the most part been extant for some years past, but information relating to them is scattered in various Blue Books, technical and scientific periodicals, and textbooks which are not ordinarily found in the library of the analytical chemist. Certain modifications, having for their object greater ease and certainty of manipulation of the apparatus used, and the attainment of a higher degree of accuracy in the analysis, have, moreover, been introduced since the original descriptions of the methods appeared. The results of the extended experience, which has now been gained, of the working of most of the methods can also be put on record with advantage. 
The methods about to be described deal only with the estimation of moisture, carbon dioxide, methane (fire-damp) or other combustible gas, oxygen, and, by difference, nitrogen (including argon) in air. The estimation of any one of these constituents to a high degree of accuracy is now required of the analyst for the purpose of controlling the efficiency of the ventilation of factories, schoolrooms, public buildings, tunnels, mines, and submarines, etc., as well as the effect on the composition of the air of rooms of different modes of lighting, heating, or artificially varying the degree of humidity. The estimation of other constituents, such as argon, of minor importance from the technical standpoint, will not be dealt with in this paper.

The methods have been, speaking broadly, devised or perfected, either independently or with the aid of collaborators, by J. S. Haldane, M.D., F.R.S., whose attention was first turned to the analysis of air in the course of numerous physiological investigations, for which methods of analysis combining extreme sensitiveness with rapidity of execution were absolutely essential. The general principles and procedure involved in these methods have proved of very wide applicability and of great utility in air and gas analysis.

The constituents of air under consideration may, with the exception of moisture, all be estimated in the laboratory on a comparatively small sample of air, which may be taken by anyone capable of following a few simple directions. (The estimation of moisture necessarily involves the use of apparatus on the spot, and, in so far as the chemical or direct methods of estimation are concerned, involves the attendance there of someone more or less skilled in the manipulation of analytical apparatus.) The method of sampling is as follows :

A narrow-mouthed bottle, of about 2 ounces capacity, with well-fitting glass stopper, is washed and dried. The stopper is greased with vaseline to insure an air-tight tit. A sample of air is taken thus: The bottle is held in one hand, and a piece of rubber tubing, about $\frac{3}{16}$-inch bore and 2 feet long, is taken in the other, and one end put in the mouth. The bottle is held at arm's length, the stopper is removed and held by the forefinger and thumb of the other hand, while the free end of the rubber tubing is passed through the mouth of the bottle to the bottom. A full inspiration of air is taken by the mouth through the rubber tube, thus drawing the air of the room into the bottle and displacing that already there. As a man, on the average, draws about 500 c.c., or 18 ounces, of air into the lungs at one normal inspiration, it will be seen that the air contained in the sample bottle is displaced nine times-or many more with a deep inspiration-by this operation. When the inspiration is nearly finished, the rubber tube is removed from the bottle, and the stopper is inserted and turned round in the neck of the bottle to distribute the vaseline evenly in the joint. A stout rubber band is passed over the head of the stopper and the bottom of the bottle, and is secured in place by affixing a gummed label round the bottle. The stopper should have a square head, to reduce the risk of the band slipping off it.

If the bottle containing the sample has to be transmitted to a distant laboratory, it may be safely sent by post in a wooden box lined with corrugated paper. Boxes divided into lined compartments for six sample bottles have been freely used for 
some years by the Factory Department of the Home Office, and also by other Government Departments, for the transmission of air samples by post. The risk of breakage and consequent loss of the sample is small: of the last 2,000 samples which have come into the author's hands after transmission by post (letter post as a rule) from various places in Great Britain and Ireland, four bottles only have been broken, and four cracked so slightly that it was doubtful if there had been any leakage of the contents. One of the travelling boxes, with the lid partially opened, displaying two bottles in position, is shown in Fig. 1 (incidentally in use as a stand for the mercury trough referred to later). In front of the box is one of the sample bottles, with rubber band and label in position after a sample of air has been taken in it.

In regard to the method of sampling, it might be contended that unskilled persons might unintentionally pass expired air directly into the bottle instead of only drawing the air for inspiration through it. Such an accident would, however, be readily detected on analysis of the sample, as expired air contains about 4 per cent. of carbon dioxide, or, say, 20 to 100 times as much as would ordinarily be found in the air of occupied rooms. Out of some 9,000 samples taken by a large number of different people of both sexes, which have come into the author's hands, there have only been two cases in which the sampling appeared to be faulty-viz., one batch of six samples, with which results of about $2 \frac{1}{2}$ per cent. of carbon dioxide indicated clearly that the samples were substantially expired air, and a single sample in which also the proportion of carbon dioxide was otherwise inexplicably high. The trustworthiness of the method has been proved time after time by concordant results having been obtained from samples taken in duplicate at the same time and spot. The samples undergo no change through lapse of time provided the containing bottles are dry and clean. If the bottles are wet but clean, carbon dioxide is liable to be absorbed from the sample ; if they are wet and contain dust, carbon dioxide is liable to be formed by bacterial action. In the ordinary course of the author's work the bottles after use are thoroughly washed, and then dried at a high temperature on a hot plate, and as soon as they are cool the greased stoppers are inserted. Thus the bottles supplied for sampling are not only dry and clean, but to all intents and purposes sterilised also.

The Estimation of Moisture and Carbon Dioxide Gravimetrically.-Provided momentary changes in the composition of the air at a given spot are not in question, the gravimetric method carried out in the manner about to be described is the most accurate and satisfactory of all chemical or physical methods of determining the proportion of moisture and carbon dioxide in air. It, however, requires the attendance on the spot of a trained analytical chemist, and hence it is used only to check the accuracy of other and more generally convenient methods, or in special cases where extreme precision is desired. On occasion also it is, for another reason, practically indispensable, in that the air of a hall, theatre, church, or debating chamber, in which the presence and operations of an analyst are undesirable at the time, can by its employment be sampled and the carbon dioxide determined unknown to the occupants. Composition gaspipe of small diameter is previously run from a suitable point or points in the hall to the analytical apparatus placed in 
an adjacent room, and thereby at any desired time during the occupation of the hall air may be aspirated through the pipe by the chemist in charge of the apparatus. This plan was followed by the author in 1902, when the air was drawn from five different points of the debating chamber of the House of Commons during sittings of the House (vide Journal of Hygiene, 1903, 3, 486; and the Blue Book, "Report, House of Commons: Ventilation," 227 of 1903). In this case the historic rule against the admission of strangers to the floor of the House during its sittings practically precluded the use of any other method of sampling and analysis, but it is obvious that in many other cases it may be considered that the operations of an analyst in a hall, etc., would disturb the occupants. The gravimetric method of determining moisture in air was recognised by Regnault in 1853 as superior in accuracy to any other, but he found it too troublesome and tedious for frequent use (Annales de Chim. et de Phys., 1845, 15, 152, and 1883, 37, 257). Shaw, in 1888, made an investigation by which he found that a $\mathrm{U}$-tube filled with pumice and sulphuric acid only absorbed moisture completely from air when the rate of aspiration did not exceed 8 to 10 litres per hour, while the tube varied considerably in weight from hour to hour more or less unaccountably. As a determination required about one hour's aspiration, there was considerable uncertainty as to the accuracy of the results obtained (Phil. Trans., 1888, A, p. 73). Haldane and Pembrey in 1890 described improvements which they had made in the method (Phil. Mag., 1890, 306). They reduced the accidental variations in the weight of the absorption tubes, and at the same time increased the efficiency of the latter (thereby rendering a more rapid rate of aspiration possible) by altering their form and weighing them against a counterpoise consisting of a tube similar in shape, size, and contents. The absorption tubes originally used by them were 4 inches deep, and 1 inch in diameter, made of thin glass, fitted with a doubly-bored cork through which an inlet pipe extended nearly to the bottom of the tube and an outlet pipe to just below the cork. The tubes were made up in pairs, the outlet pipe of one and the inlet pipe of the other being formed of one piece of glass by bending at the top. The insertion of the long inlet pipe in the tube after it had been charged with the absorptive material was rather troublesome, and the doubly-perforated cork increased the risk of leakage. To overcome these defects absorption tubes were later blown in pairs, with the inlet and the outlet tubes blown into the walls about $\frac{3}{4}$ inch below the top. Thus after charging, only well-fitting shallow plain corks had to be inserted. The corks, about $\frac{1}{2}$ inch deep, were soaked in melted paraffin wax, and pressed into the cleaned and warmed mouths of the tubes until they were a little below the tops. A layer of paraffin wax was then spread over the cork to the level of the top of the tube. Before each time of use each tube was tested for soundness by connecting its inlet to one limb of a $\mathbf{U}$-shaped water-gauge, slightly exhausting through and then closing its outlet, and observing whether the water in the gauge remained at the same level for about half an hour. When not in use the tubes were closed by stoppers of about $\frac{3}{4}$ inch of rubber tubing closed at one end by a short piece of glass rod. The counterpoise served, of course, for any number of absorption tubes in use at the same time. The stoppers were removed simultaneously from all the tubes and the counterpoise before weighing, and replaced simultaneously when 
all the tubes had been weighed. This form of double absorption tube has been largely used by the author, and has given very satisfactory results. It was obvious, however, that ground-glass stoppers would be superior to the corks, but it was not forthwith apparent how they could be fitted without interfering with the principle involved in the design of the tubes. Ultimately, however, a stoppered form of this type of absorption tube was evolved in B. Blount's laboratory (about 1904), and this, with a slightly wider stopper, is now being used by the author. (An illustration, to scale, of this tube, with, however, the inconveniently narrow stopper, has been given in vol. $i$. of the third and fourth editions of the author's "Handbook on Gas Manufacture.")

The reasons for the adoption of the Haldane and Pembrey type of absorption tube, and for weighing it against a similar counterpoise, may be briefly enunciated. The amount of moisture on the outer surface of such tubes varies from time to time; it is diffecult absolutely to exclude penetration of moisture to the inside, and variations of temperature and barometric pressure may affect the weighings. These variations, which in the aggregate may cause a tube to vary more than $2 \mathrm{mgm}$. in weight, are rendered of no effect by the practice of weighing the tube against a similar tube which has been throughout exposed to the same conditions. The form of tube is chosen with a view to securing, weight for weight, great absorptive efficiency. Owing to the great width of the tube the air passes upward through the absorptive material at a comparatively low rate of flow, thus affording time for absorption. The sulphuric acid on the pumice is gradually diluted by the moisture absorbed from the air, and ultimately dilute acid collects in the bottom of the first of the pair of tubes, and continues to do preliminary absorptive work on the air without impairing the strength of the overlying pumice in the same tube or of the pumice in the second tube. In the ordinary $U$-tube, on the other hand, the diluted acid would spread throughout the first limb and the bend, and only the pumice in the second limb would retain its full strength for any length of time. One double absorption tube charged with sulphuric acid pumice weighs 70 to 80 grams, and will continue to absorb moisture completely from air even after 12 grams of moisture have already been absorbed-that is to say, after 2,000 litres or more of air have been aspirated through it. The rate of aspiration may be as high as 7 litres per minute, and as 5 to 10 litres is a convenient quantity for one determination, it will be seen that the moisture in air may, if required, be accurately determined gravimetrically over as short a period as two minutes. The method thus becomes invaluable as a check on the readings of wet and dry bulb hygrometers or other psychrometers with which instantaneous readings are made. The author proposes to deal in a subsequent communication with the comparative results afforded by certain of these instruments as well as by a volumetric absorption apparatus somewhat resembling one described by S. Rideal last year (Journ. Royal Sanitary Inst., 1908, 39, 87).

The advantages of the Haldane-Pembrey type of double absorption tube are even more marked when it is applied to the absorption of carbon dioxide. The first of the paired tubes is then filled with uniformly granulated soda-lime (granules 1 to $2 \mathrm{~mm}$. in diameter) free from dust, and the second with pumice saturated with strong sulphuric acid. The soda-lime absorbs carbon dioxide from air aspirated 
through the tube, while the moisture thereby displaced from the soda-lime is retained by the sulphuric acid. The net increase in weight of the double tube, therefore, gives forthwith the amount of carbon dioxide absorbed. It is, of course, necessary that the air entering the soda-lime should be free from moisture, and consequently a double absorption tube containing sulphuric acid pumice is placed before it, and the moisture in the air may thereby incidentally be determined simultaneously with the carbon dioxide. As the absorption of carbon dioxide by soda-lime is less vigorous than that of moisture by sulphuric acid, it becomes necessary, however, to aspirate at a somewhat lower rate, and it is desirable to have a double guard tube charged with soda-lime and sulphuric acid pumice to detect any failure on the part of the first soda-lime tube to absorb the whole of the carbon dioxide. The train of apparatus is therefore set up in the following order, starting at the air intake: (1) A double absorption tube, charged throughout with sulphuric acid pumice, for the absorption (and determination if desired) of the moisture. (2) A double absorption tube, the first tube of which is charged with soda-lime, and the second tube with sulphuric acid pumice. This tube ordinarily absorbs all the carbon dioxide from the air. (3) A double absorption tube similar to the last, acting merely as a guard tube. And (4) an aspirator of 10 to 50 litres capacity.

The rate of aspiration should not on the average greatly exceed 1 litre per minute. At 2 litres per minute a trace of carbon dioxide will usually reach the guard tube. For outside air as much as 20 litres should, if possible, be aspirated, as the increase in weight of the absorption tube would with that quantity of air be only about $12 \mathrm{mgm}$. The absorption tubes are, of course, weighed against a similar tube as a counterpoise. It will thus be seen that a determination ordinarily covers a period of about twenty minutes, but for vitiated air ten minutes will suffice. On the other hand, the time may be extended so that the determination gives the average amount of carbon dioxide in the air over a period of, say, one hour. The prolongation of the test is limited practically only by the capacity of the aspirator, which may, of course, be of a double vessel reversible type. The author has used carboys, suitably fitted with a cork and siphon-tube, as aspirators up to a capacity of about 55 litres. The capacity of the aspirator, from a mark on the neck, to which it is filled, to a mark near the bottom, should be accurately determined; and if it is desired to stop a test before the latter mark is reached, it is easy afterwards to measure the quantity of water remaining down to that mark. It is often convenient to adjust the lower mark to the position for a given round number of litres, and so reduce trouble in calculations. An approximate scale of litres on the side of the aspirator is an aid in adjusting the rate of aspiration. In all cases a thermometer must be fixed in the aspirator and read when the aspirator is stopped, and the height of the barometric column at the time of the test must be noted, so that the volume of air collected in the aspirator may be corrected for deviation from its volume under normal temperature and pressure. Unless the room in which the aspirator is used varies little in temperature, and the water is at the temperature of the room, it is advisable to jacket the aspirator - with felt, for instance. It must also be remembered that the volume measured by the aspirator is air saturated with moisture, but deprived of carbon dioxide. The amount of the latter absorbed by the soda-lime must be added to the 
aspirated volume of air in calculating the exact proportion of carbon dioxide in the air examined. The moisture correction must be similarly made if it is desired to refer the results to dry air, or to air of the degree of humidity of that examined.

Simultaneous determinations by the gravimetric method as here described will always give results agreeing as to moisture well within 1 per cent. if only 6 litres of air are taken, and as to carbon dioxide within 2 per cent. if only 20 litres of air are taken. The mean error is considerably lower-viz., about 0.1 per cent.-and as the unavoidable differences in weighings are chiefly responsible for any want of concordance in analyses made in duplicate, it is obvious that by increasing the volume of air aspirated the degree of accuracy of the results may be enhanced to almost any desired limit. That is to say, if the air of a room contained exactly 5 volumes of carbon dioxide per 10,000 volumes of air, determinations could be made by this method, of which the extreme results would be 5.1 and 4.9 volumes. If the air contained exactly 20 volumes of carbon dioxide per 10,000 , more than $20 \cdot 1$ or less than $19 \cdot 9$ volumes should not be found by this method. Ordinarily, with reasonable care in working, the deviation would be considerably less, as the mean error of a carefully made determination is only about \pm 0.01 volume of either moisture or carbon dioxide per 10,000 volumes of air.

The Estimation of Carbon Dioxide Volumetrically.-The author thinks it necessary on this occasion only to refer to the volumetric method introduced by J. S. Haldane about 1900 (vide Journ. of Hygiene, 1901, 1, 109; and the Blue Book, First Report of the Committee on the Ventilation of Factories and Workshops, Cd. 1302 of 1902). It is tolerably well known, so that only a brief description need be given, and certain modifications of the apparatus used, which the author has found convenient, referred to. It depends on the absorption of the carbon dioxide in a measured volume of gas by solution of caustic potash. The apparatus used comprises a burette of about 20 c.c. capacity, of which the stem represents a little over 0.2 c.c., and the bulb the remainder. The stem is graduated in 100 divisions, each representing $\frac{1}{10 \delta \delta \sigma}$ of the capacity of the burette from the bottom (zero mark) division on the stem to the tap above the bulb. One division is about 0.04 inch, or $1 \mathrm{~mm}$., in length, and it is possible, therefore, to read the height of the column of mercury in the stem to one-fifth of a division, or to $0 \cdot 2$ part per 10,000, with fair precision. The changes of volume of the portion of air enclosed in the burette, resulting from variations of temperature and barometric pressure, would frequently be greater than the reduction of volume due to the absorption of the carbon dioxide, and would thus ordinarily prevent this reduction being directly observed. A change of temperature of one-tenth of a degree Fahrenheit represents approximately the total change of volume due to the absorption of the proportion of carbon dioxide ordinarily present in outside air. It would thus be practically impossible to make readings of temperature with sufficient accuracy for correcting the volume of air in the burette to normal. Fortunately, this trouble may be avoided, and a higher degree of accuracy attained by the employment of a control tube of the same size and shape as the burette placed alongside the latter in a water-jacket. The stem of the control tube is filled with water, so that the air within the tube is saturated with moisture, and a trace of water in the burette serves similarly to saturate the sample of air enclosed therein 
Referring to the illustration of a modified form of this apparatus in Fig. 1, it will be seen that the burette $A$ and control tube $B$ communicate by means of independent connections of about the same capacity with separate limbs of what is in effect a U-tube or differential pressure gauge, $\mathrm{C}$, charged with solution of caustic potash. The limb in communication with the burette is enlarged, below the normal surface-level of the solution, to a bulb, $D$, of about 20 c.c. capacity, and a tubulure at the base of the $\mathrm{U}$-tube communicates by flexible tubing, $\mathrm{E}$, with a glass bulb, $\mathrm{F}$, which may be raised or lowered over a limited range by sliding its stem in the spring-

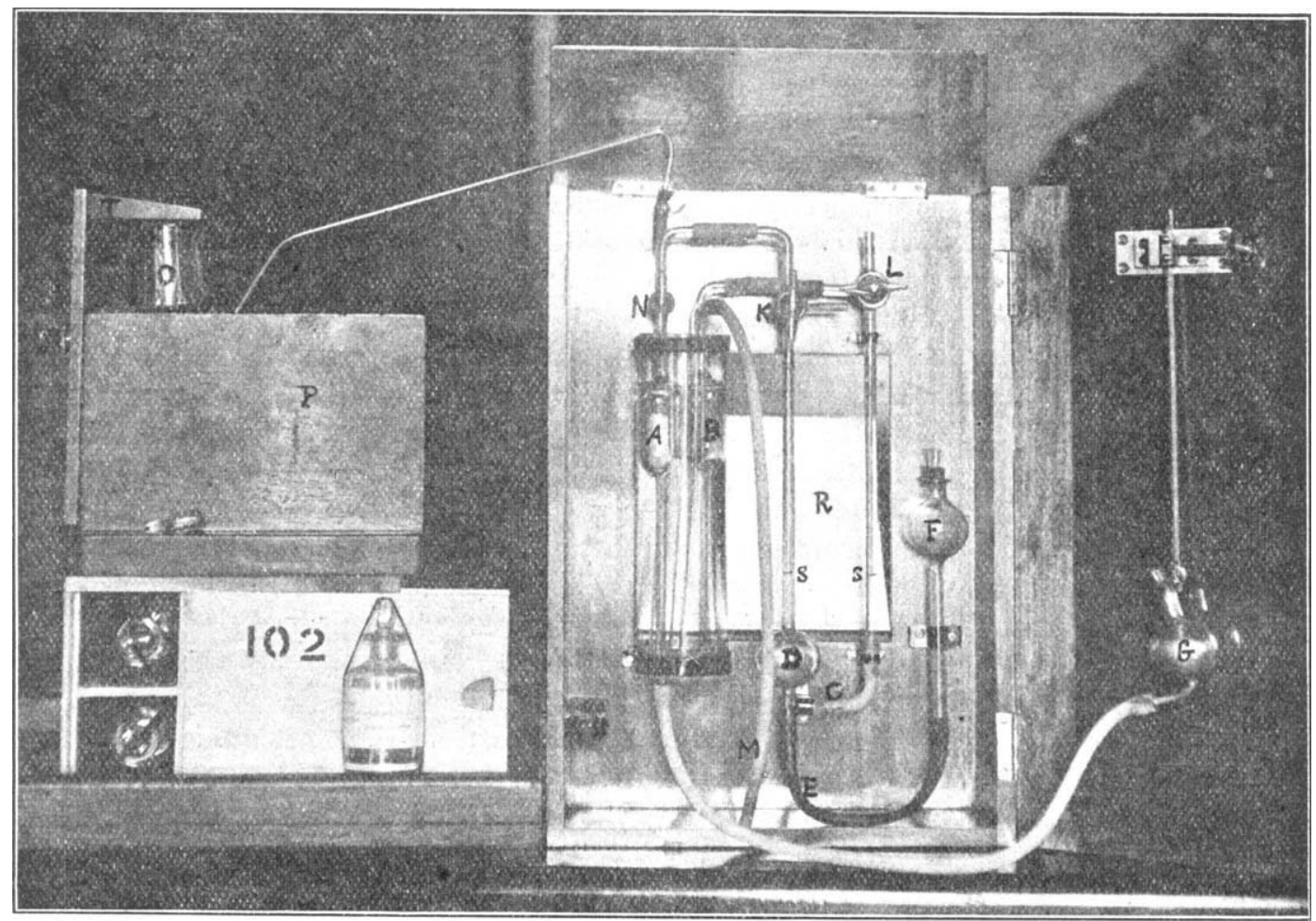

Fig. 1.-Apparatus for the Determination of Carbon Dioxide in Air.

clip which supports it. The lower end of the stem of the burette communicates, by means of flexible tubing, with a reservoir, G, containing mercury. This reservoir is carried by a rack, $\mathrm{H}$, which is raised and lowered by turning a milled head, $\mathrm{J}$, attached to the spindle of the pinion on which the rack travels. By means of three-way taps, $\mathrm{K}$ and $\mathrm{L}$, on the connections, both the burette and the control tube may be put out of communication with the U-tube and into communication with the outer air. The pressure in both may thus be equalised with that of the outer air. After this equalisation, if they are shut off from the outer air and put in communication with the potash solution in the $U$-tube, the solution will stand at approximately the same 
level in both limbs of the tube. If now the water in the water-jacket is agitated by blowing through the tube $\mathrm{M}$ from time to time to preserve uniformity of temperature throughout it, and, consequently, uniformity of temperature of the burette and control tube, the level of the potash solution in the two limbs of the U-tube will be affected equally by changes of volume of the air contained in the equal-sized burette and control tube arising from variations of temperature or atmospheric pressure. Hence so long as the potash solution in the two limbs of the U-tube is kept at the same level, any observed alteration of volume of the air contained in the burette is due to an alteration of mass, and not to mere expansion or contraction of the original mass of air. The means is thus obtained of measuring accurately the amount of any constituent of the air which is removed therefrom by the action of any absorbent. The carbon dioxide in air is thus estimated by passing the measured volume of air to and fro between the burette $\mathrm{A}$ and the caustic potash solution in the bulb $\mathrm{D}$ in the one limb of the U-tube. The mercury reservoir $G$ is raised and lowered to effect this transference. The volume of air is measured again, with the levels of the potash solution balanced in the two limbs, and the reduction in volume since the first measurement shows the volume of carbon dioxide absorbed from the known volume of air. As the volume of air taken for an estimation in the apparatus in question is always about 10,000 times that contained between two adjacent graduations on the stem of the burette, readings of the level of the mercury in the latter, made in the conditions indicated, before and after passing the contained air to and from the potash solution twice or thrice, show directly by difference the volumes of carbon dioxide per 10,000 volumes of the air represented by the sample. The expression "U-tube " has been used in the foregoing explanation of the function of the control tube because its purpose is that of a $U$-gauge, by which any difference between the pressures prevailing in the two limbs may be at once detected. As the limbs of the gauge are in contact with confined volumes of air, an alteration of one volume independently of an alteration of the other may be readily observed and measured. As a fact, the actual form given, for other reasons, to the parts here referred to as a $U$-tube does not at once suggest that they form a $U$-pattern differential pressure gauge.

The use of a control tube in gas analysis was described by Williamson and Russell in 1868 . They, however, used with it a mercury gauge, which, of course, was far less sensitive than the gauge described above, in which the liquid is a 10 per cent. aqueous solution of caustic potash.* Pettersson subsequently used a drop of oil in a horizontal tube as a gauge to indicate difference of pressure between a burette and a control tube. The introduction of the simple gauge, containing solution of caustic potash, which serves both as a gauge liquor and as an absorbent for carbon dioxide, is due entirely to J. S. Haldane. It is applied with advantage, as will be seen later, in apparatus for the estimation of other constituents in air, and is naturally also applicable to many estimations of constituents of industrial gases. The use of the control tube and differential gauge, containing an aqueous liquid, has led to a degree of precision, combined with rapidity of work, in air analysis that otherwise is quite unattainable.

* F. R. Japp, in 1891, used a control or " regulator" tube with a mercury differential gauge in his "gravivolumeter" (vide S. Rideal [infra] and Journ. Chem. Soc., 1891, 59, 894). 
The Haldane apparatus for the volumetric estimation of carbon dioxide in air is in its original portable form perfectly convenient for estimations carried out in situ. For use in the laboratory, when the sample of air for analysis is drawn from a bottle inverted over mercury in a trough, the author has tried modifications with a view to rendering the burette more readily accessible for cleaning, and the three-way tap at the top of the burette less liable to obstruction by a globule or plug of mercury (or even of water). Such a globule sometimes interferes with free communication between the potash solution and the burette, and so with the proper action of the differential pressure gauge. The author now prefers a tap, $N$ (Fig. 1), with two parallel ways, by one of which the sample is drawn into the burette from the bottle $\mathrm{O}$, inverted in the mercury trough $\mathrm{P}$, and by the other of which the burette is put in communication with the potash solution. Mercury need then never enter the latter way through the plug of the tap, so that there is no risk of obstruction of this way. A three-way tap, $K$, is placed in the connection between this tap and the potash solution, so that either the latter or the burette may be readily put into communication with the outer air. The apparatus may still be in a portable cabinet, as shown in the figure, but as at times it is more convenient to work with the light in front of, instead of behind, the observer, a flap, $R$, has been arranged in the back of the cabinet, through the opening of which light may reach the stem of the burette and the levelling marks $\mathrm{S}, \mathrm{S}$ on the differential gauge. The flexible rubber connection between the differential gauge and the potash reservoir F may, when new, and if it contains much sulphur, form a sulphur compound with the solution of potash, which then absorbs some oxygen as well as carbon dioxide. This action of the potash on the rubber soon ceases, and gives no trouble after the potash solution has been twice renewed after standing in the apparatus for about a week each time.

Consecutive estimations of carbon dioxide by the Haldane volumetric apparatus should give results always agreeing within 1 volume in 10,000. The normal deviation from the mean is, however, only about \pm 0.2 volume, which is about the limit of accuracy of readings with the naked eye of the graduations of the stem of the burette; that is to say, a sample of air containing exactly 20 volumes of carbon dioxide per 10,000 would by this apparatus, with careful manipulation, not appear to contain more than 20.2 or less than 19.8 volumes. The 2-ounce bottles used for samples contain sufficient air for three estimations, and it is the author's practice always to make two estimations, and if the results differ appreciably, to make a third.

When large numbers of samples of air are dealt with, it is convenient to have a mercury trough of as limited capacity as is consistent with comfortable manipulations. The author uses wooden troughs (P, Fig. 1, and A, Fig. 3) with the reservoir narrowing towards the base, and provided with a raised flange to hinder overflow of the mercury when the fingers are plunged in for removing the stopper of the bottle. A cover, B (Fig. 3), serves to keep out dust when the trough is not in use, and also to prevent the mercury being splashed over by slight jars to which its stand, C (Fig. 3), may accidentally be subjected. The light wooden arm T (Fig. 1), lightly clamped by the screw $U$, serves to hold the bottle, after inversion and removal of the stopper, steady in the trough.

The Estimation of Methane (Fire-Damp) and Oxygen Volumetrically.-The 
estimation of methane (fire-damp) in the air of mines, or of small proportions of a combustible gas in air generally, is best carried out by the combustion of the methane or other combustible gas by means of the oxygen contained in the sample of air. The combustion is effected by passing the air containing the combustible gas to and fro over an electrically-heated spiral of fine platinum wire. This method of effecting the complete combustion of small quantities of methane was first proposed by Coquillion (Comptes Rend., 1877, 84, 458). It avoids any need for the addition of hydrogen, detonating gas, or neat oxygen to the air under analysis, which addition is necessary when explosion by a spark is relied upon for the combustion. By its use complete combustion may be absolutely insured. If the proportion of methane exceeds about 8 or 9 per cent. [the precise proportion depending on the amount of black damp (carbon dioxide plus nitrogen) also present], an addition of a measured volume of pure air only will be necessary to effect the complete combustion of the methane. No neat oxygen is required even with such an abnormal sample of mine air. The platinum spiral is fixed in a suitably shaped pipette, D (Figs. 2 and 3), in a manner admitting of its being readily replaced in case it is fused by too strong a current. In the apparatus used by the author it requires from 2 to 4 volts to raise it to incandescence, and this is readily supplied from an accumulator, E (Fig. 3), through a variable resistance, $\mathrm{F}$, by means of which the wire may be gradually brought to the desired degree of incandescence. The contraction due to the combustion of the methane is measured, and then the carbon dioxide produced is absorbed in the caustic potash solution in the pipette $G$, and its volume ascertained by difference. If the volume of carbon dioxide is other than half the contraction, the combustible gas is not wholly methane. If methane and only one other combustible gas, such as carbon monoxide, are present, the amounts of methane and carbon monoxide may be calculated from the ratio of the contraction to the carbon dioxide resulting from the combustion. In samples of air from coal-mines, however, the combustible gas present is almost always pure methane, and the carbon dioxide produced tallies accordingly with the contraction on combustion over the incandescent platinum spiral. It is necesssary, of course, that the carbon dioxide in the sample of air should have been absorbed by the caustic potash solution in the pipette $G$ prior to the combustion of the methane. After combustion of the methane, the residual oxygen in the sample is absorbed by means of the alkaline solution of pyrogallol contained in the pipette H. Oxygen equivalent to double the volume of methane found must also have been present, and consumed in the combustion of the methane. This must be added to the volume of oxygen absorbed by the pyrogallol solution in order to obtain the total volume of oxygen in the sample. As a check on the working, a second portion of the sample may be afterwards taken, and after absorption of the carbon dioxide by caustic potash solution, the total oxygen may be at once absorbed by the pyrogallol solution. The proportion of oxygen thus found should tally with that already computed by adding the volume of oxygen used for the combustion of the methane to the volume of residual oxygen found after that combustion. A check on the whole analytical work is thus readily obtained.

The pyrogallol solution for the absorption of oxygen is prepared by adding 100 c.c. of a completely saturated solution of caustic potash to 10 grams of 
pyrogallic acid contained in a stoppered bottle. After shaking and allowing a little time for the pyrogallic acid to dissolve, a sufficient quantity of the solution to fill the absorption pipette $H$ is transferred to the latter by means of an ordinary measuring

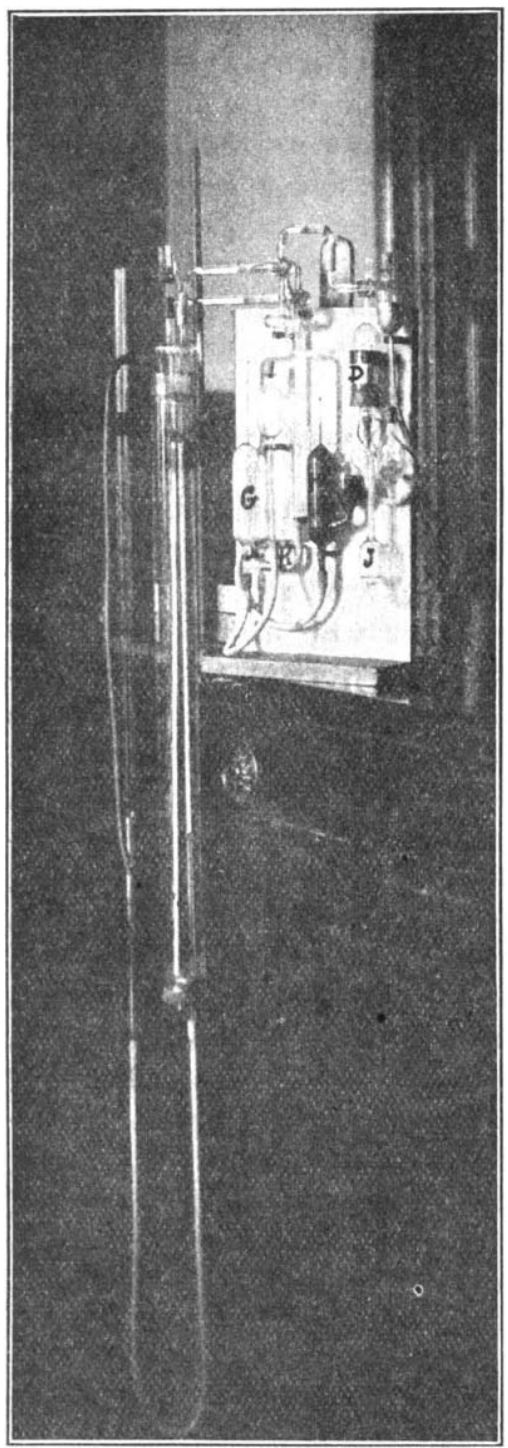
pipette. The outer bulbs $J$ of the double absorption pipette are then charged with strong solution of caustic potash, to prevent the oxygen and moisture of the atmosphere gaining access to the pyrogallol solution. An absorption pipette thus charged should absorb oxygen rapidly and certainly for 150 to 200 analyses of air. The absorption is rapid and complete, and no trace of carbon monoxide is formed. Proof of the latter statement is easily obtained by passing the residual gas, together with a fresh proportion of air, over the heated platinum spiral. No contraction or formation of carbon dioxide will be detected. If, however, the solution of pyrogallol has been prepared with a weak solution of caustic potash, the absorption of oxygen is attended by the formation of carbon monoxide.

The burette used for the estimation of methane and oxygen has a stem of rather more than three-tenths of the total capacity. The stem is graduated in divisions each $\frac{1}{1000}$ (Fig. 3) or $\frac{1}{2000}$ (Fig. 2) of the total capacity, and it is possible to read to one-tenth of a division without the use of a telescope. Thus, estimations of methane and oxygen may be made with certainty within 0.01 volume per 100 volumes of air.

Careful calibration of the burette used is essential in all cases. A determination of the oxygen in pure country air will disclose any error of moment in the burettes described, and if the result differs from 20.93 per cent. by more than \pm 0.01 , other analytical results should be corrected accordingly. But if careful calibraFig. 2.-Laboratory APPARATUS FOR the tion of the burette with mercury shows that DETERMINATION OF CARBoN DIOXIDE the stem is irregularly divided, the burette METHANE (OR UTHER COMBUSTIBLE GAS)
AND OXYGEN IN THE AIR.

This apparatus is shown in two sizes, one being in a cabingt (Fig. 3) and readily portable, the other (Fig. 2), having a larger and longer burette, being 
suitable only for laboratory use. The larger apparatus has a somewhat lower limit of error than the smaller, but the latter can be used with careful manipulation to the degree of accuracy already stated. With the portable apparatus, the capacity of the burette is about 10 c.c ; with the larger, about 20 c.c. The large number of analyses of samples of mine air made, some by the author and some by Mr. E. B. Whalley, for the Royal Commission on Mines, of which the results are recorded in the recently issued Blue Book (Cd. 4551), containing reports on "The Ventilation of Coal-Mines and the Methods of Examining for Fire-Damp," by

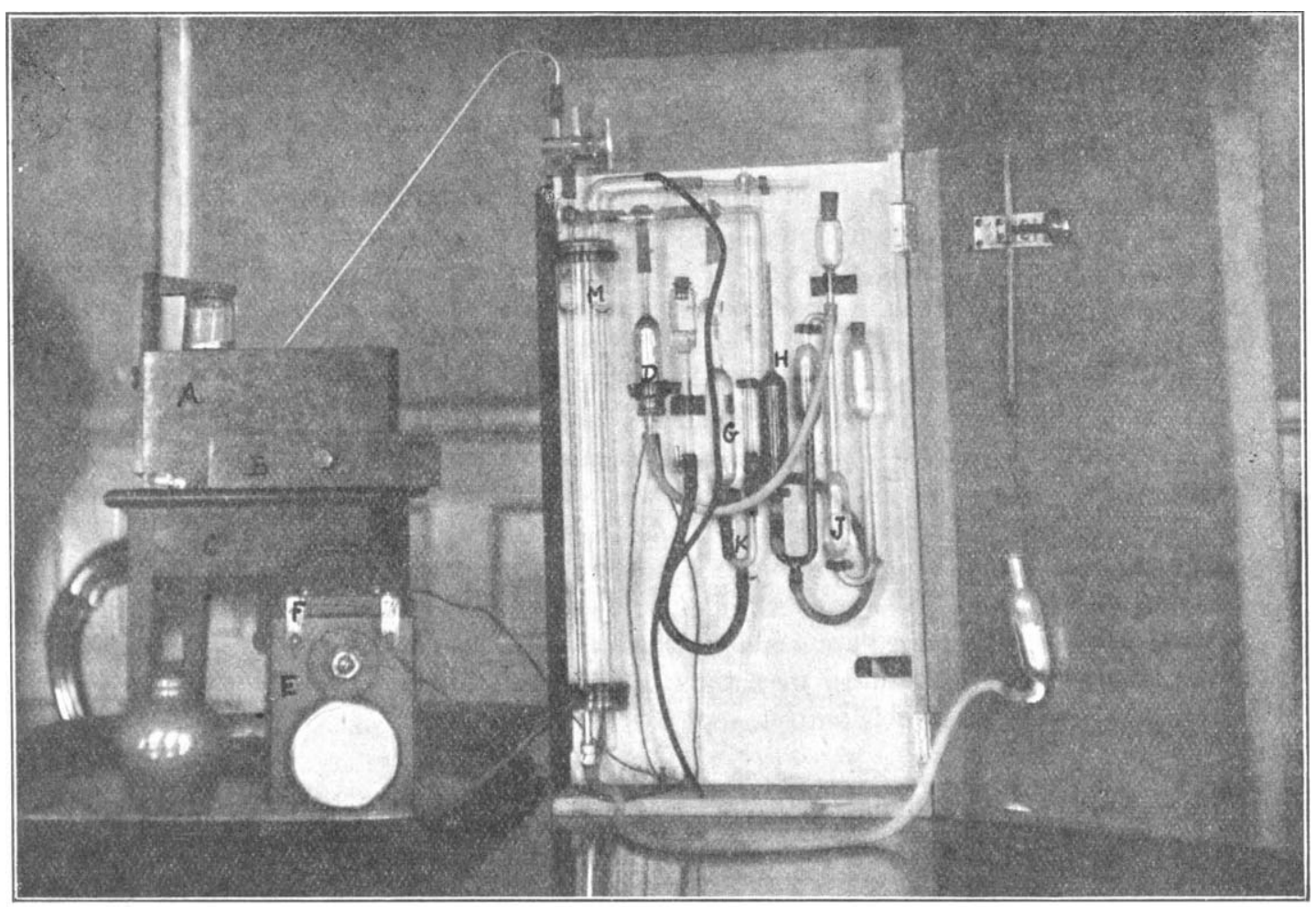

Fig. 3.-Portable Apparatus for the Determination of Carbon Dioxide Methane (or other Combustible Gas) and Oxygen in Air.

Professor John Cadman and Mr. Whalley, were made with these two types of apparatus. The smaller apparatus was originally described by J. S. Haldane (Journal of Hygiene, 1901, 6, 74), and the larger in the textbook on " The Investigation of Mine Air," by Sir C. Le Neve Foster and J. S. Haldane, but some small modifications have been introduced in the apparatus as now illustrated. It will be observed that the differential pressure gauge, containing 10 per cent. solution of caustic potash, is embodied in both (K, Figs. 2 and 3), as well as the control tube M immersed alongside the burette in a water-jacket. It is necessary, before com- 
mencing an analysis, that the connections between the burette and the absorption pipettes should contain only nitrogen. This is the residue naturally remaining after a complete analysis of a sample of air; consequently analyses may be made one after another without intermediate delay. When, however, the apparatus has been put aside for twenty-four hours or longer, a certain amount of oxygen may have found its way into the connections by transfusion through the rubber joints, and this must be absorbed in the pyrogallol pipette before an analysis is started. If before the apparatus is put aside after use the residual nitrogen in the burette is transferred to the pyrogallol pipette, and enclosed in the latter by turning the tap, this nitrogen may be employed when the apparatus is next brought into use to displace air which may have found its way into the connections. As a fact, if stout-walled rubber tubing of good quality is used for making the joints, and it is lightly greased inside with vaseline, very little oxygen finds its way into the connections, even after prolonged disuse.

The time required for the determination of carbon dioxide and either methane or oxygen is about fifteen minutes, or for all three constituents half an hour. The limit to the employment of this type of apparatus for the analysis of more complex mixtures of gases is fixed in practice by the number of pipettes required, since each connection previously used in the course of the analysis must have its gaseous contents displaced at each stage in order that each constituent may be absorbed from the whole of the volume of original gas taken for analysis. Four pipettes may be used with advantage for certain gaseous mixtures, but if more than four are required the process of analysis becomes very tedious with this type of apparatus. It is often possible, bowever, by a judicious disposition of the taps and connections, and by omitting the determination of some one constituent, having no significance for the work in hand, or by determining two constituents together, to render the type of apparatus described applicable to the analysis of relatively complex technical gaseous mixtures. Advantage may thus be taken generally of the high degree of accuracy which is attainable with it.

\section{Discussion.}

The Chalrman (Mr. Blount) said that one of the many interesting and important points brought out in this paper was that the old cumbrous methods of determining the constituents of a gas were dead. Nowadays, if the constituents. of a gas were known generally, it could be readily analysed in a rapid and very accurate manner. No doubt, in the case of a perfectly new gas or a new kind of mixture, the methods of Bunsen and Frankland would have to be resorted to; but with some knowledge of the probable composition of the gas, much simpler and more precise methods could be used. Another point was that compensation for variations in temperature and pressure had been applied in both the gravimetric and the volumetric forms of this process, thus eliminating errors which otherwise were liable to become hopelessly large, having regard to the limits of accuracy which it was necessary to attain. In saying this he was not speaking merely academically, for, through the kindness of Dr. Haldane and Mr. Butterfield, he had been greatly helped when on some occasions he bad had to analyse the air 
of halls and theatres; and the small contribution which he had been able to make in designing, with the help of his colleagues, a tube suitable for the absorption of carbon dioxide was but a trifling return for the benefit he had obtained from that help. He had been much struck by the way in which, while complying with all requirements as to precision, the apparatus and the method of collecting samples had been simplified, and he thought that Mr. Butterfield and bis coadjutors were to be heartily congratulated on the perfection they had attained in this important branch of analysis.

Mr. A. E. Parkes asked if any steps were taken to exclude dust and carbon dioxide in the moisture determination, or whether the influence of these was negligible; and also whether, when the samples were taken in an adjacent chamber, after the air had been drawn through metal piping, there would not be some risk, if the air was fairly saturated, of moisture being condensed in the pipe. He thought it would be interesting if Mr. Butterfield would describe a little more fully the method of transferring the samples from the small bottles to the apparatus. At the time when the tunnel under the Thames from Stepney to Rotherhithe was being made, he (Mr. Parkes) had had to analyse samples of the air from the point where the men were at work in compressed air in the chamber behind the shield. That air, of course, was in a somewhat abnormal condition. The samples were taken in the old-fashioned way in bottles of about 3 litres capacity, and Pettenkofer's method of estimating the carbon dioxide was used. The proportions of carbon dioxide found varied considerably-from about 4 parts per 1,000 down to as little as 0.4 to 0.5 part. The higher figures were obtained in winter and the lower in summer, this being probably due to the fact that in winter free ventilation was objected to by the men on account of the cold.

Mr. W. T. BuRgess suggested that it would be readily possible to design a simple but effective valve which would at any rate prevent blowing into the bottle. With regard to physical methods of determining moisture in air, he had recently had occasion to examine some wet and dry bulb thermometers, and had come to the conclusion that in the instruments usually sold the arrangement for keeping the wet bulb wet was not as good as it might be. Properly speaking, the wet bulb should be covered with very fine muslin tied tightly round it, and, instead of the usual large "wick," there should be two or three threads of cotton leading from the muslin into a vessel of water some 3 or 4 inches away.

Mr. G. N. HuntLy said they were indebted to the author for calling attention to the utility of the Haldane apparatus, which hitherto had been hardly known to more than a few. Some sixteen years ago he first used the compensation method, and he thought that the use of a compensating air-tube was first suggested by Pettersson; but the original device was by no means practical, and he believed Dr. Haldane was the first to work out the ingenious arrangement described in the present paper. He (Mr. Huntly) had used the small range apparatus for some time, and had found it capable of a degree of accuracy of about 1 in 20,000, but it was possible to work as closely as 1 in 50,000 under favourable conditions. In the larger apparatus the difficulty lay in the graduation of the tubes. He had found a variation, in the graduated portion only, of as much as 3 per cent. by volume ( 3 per cent. 
of 6 c.c. $=0.18$ c.c.), and many of these badly graduated tubes were to be met with. Tubes with such small divisions $(0.01$ c.c.) were difficult to calibrate, and the use of the tube wet caused variations in the shape of the meniscus, so that it was not possible to read accurately to a tenth of a division, and an accuracy of 1 in 10,000 was as much as could be really relied upon. The method of calibration described in Haldane and Foster's book on Mine Air was scarcely good enough for accurate work, as the meniscus, which could not be allowed for, amounted to about half a division. The only satisfactory way was to fuse on a three-way capillary tap, and run the mercury up from the bottom, running the whole of it out each time; but this was a very tedious operation. For a single determination, such as that of carbon dioxide, the method was an ideal one, but the addition of further pipettes for other determinations made its working less convenient. At the end of the operation all these added spaces ought in theory to be full of nitrogen, but in practice it was difficult to insure this, so that with every additional determination the accuracy fell off. Considerable difficulty arose with regard to the combustion of traces of methane. He very much doubted whether the last traces of combustible gas were completely burnt, at any rate when the original quantity was small. Complete combustion could be insured by adding some hydrogen, but the introduction of another gas was objectionable.

Dr. RIDEAL said that a compensating "gravivolumeter," as it was called, was described by Japp some years ago (J.Chem. Soc., 1891, 894), which seemed to be the forerunner of the $\mathrm{CO}_{2}$ apparatus shown. He (Dr. Rideal) had had some experience with the small portable form of the apparatus described by Mr. Butterfield, and had found that, as Mr. Huntly had mentioned, it had some disadvantages, from which, however, the larger form seemed to be free.

Mr. Bevan said that in some analyses which he had once had to make of carbon filaments for electric lamps he had found that variations in temperature and pressure caused large errors, which would be eliminated by the compensating device now described. He should like to hear if Mr. Butterfield had tried the use of silica tubes, and could say whether the quantity of moisture condensed on these was less or greater than with glass.

Dr. Lessing said that, in determining the proportion of nickel in nickel carbonyl gases (by decomposing the nickel carbonyl by heating to about $200^{\circ} \mathrm{C}$., which resulted in an increase in volume of 4 to 1 ), he had observed that, whenever air was added to the gas, or when air leaked into the tube, there was always a contraction in volume. This had not been explained, but might be due to oxidation by some nickel present in the tube. This contraction was a parallel to the abnormal expansion of methane when insufficient air was present for complete combustion.

Mr. Butterfield, in reply, said that this gravimetric mode of analysis was often used in conjunction with a bacteriological examination of the air, and in that case a tube containing sterilised cotton-wool was attached to the free end of the tube through which the air was drawn, so that the air was filtered at any rate through that. As to condensation of moisture in the metal pipe, that was liable to occur if there were appreciable differences of temperature, and he did not advocate the drawing of samples through a long tube when the moisture was to be determined 
exactly, though there was not much fault to be found with this plan in regard to the determination of carbon dioxide. As to the transference of the sample to the apparatus, the neck of the bottle was inserted in the mercury trough, the stopper taken out beneath the mercury, and the free end of a bent tube (already filled with mercury and connected to the burette) inserted through the mouth of the bottle, which was then clamped down, and the sample drawn over. In examining a number of samples in succession, it was sufficient to leave a plug of mercury in the open end of the tube, drawing over the air contained between that plug and the apparatus into the burette and expelling it into the open air, and then drawing in the sample; but the orthodox way was to fill the tube first with mercury. In examining air under pressure all sorts of difficulties might arise, and the quantity of carbon dioxide found in compressed air workings sometimes varied more or less unaccountably. One point of importance when samples were taken under considerable pressure (as, for instance, at the bottom of deep mines) was that a very stout band should be placed over the stopper, otherwise, under the lower barometric pressure at the surface, the stopper might be blown out. He had recently been using this type of apparatus with satisfactory results at considerably reduced pressures, and also at pressures considerably higher than the normal. He had hoped to deal with the subject of wet and dry bulb thermometers, or psychrometers, but the paper had already become too long. He showed, however, a specimen of the form of wet and dry bulb thermometer now generally accepted by German physicists, and known as the Assmann psychrometer. The ordinary form-i.e., Mason's hygrometer-was perfectly satisfactory when used outdoors with currents of air passing over the wet bulb; but when it was indoors the damping arrangement created round the bulbs a local humidified atmosphere which remained unchanged, and, consequently, the true humidity of the room was not shown, the air in the neighbourhood of the reservoir being saturated to a greater extent than the air of the rest of the room. The German instrument had a clockwork fan which drew the air over the bulbs, so that there was no stagnation, and there was an arrangement for moistening tine wet bulb every ten minutes-the period of the fan's running. This was now considered to be the best physical means of determining the humidity of the air, and in a future paper he hoped to compare the results yielded by this and by the gravimetric method. It might be mentioned that some years ago Dr. Pembrey had proved that the ordinary Mason's hygrometer was perfectly reliable outdoors. Dr. Pembrey's determinations extended over several months, this gravimetric method, for which he was partly responsible, being used in the comparisons. As Mr. Huntly had remarked, the gravimetric method as here shown for the determination of carbon dioxide was not very widely known, which was one of the reasons for the writing of the present paper. The accuracy, as apart from the consistency, of the results obtained with the small apparatus had been amply checked by comparison with the results of gravimetric determinations. The Pettenkofer method was, of course, a very good one, but a larger sample was required, and in order to be sure of the results it was necessary always to make a control test on outside air. The results merely showed the degree of divergence from the outside air. This, of course, was generally what was really wanted, but there was an error of uncertain magnitude and generally of uncertain origin. Mr. Huntly had remarked 
that in the application of the apparatus to gas analysis the necessity for using several pipettes made an analysis a long proceeding, because of the necessity for clearing the gas out of the connections each time. This was quite true, but with only three determinations there was not very much difficulty, it being possible to make complete and exact determinations of carbon dioxide, methane, and oxygen in half an hour once the apparatus had been prepared for use. At the outset, as was mentioned in the paper, the connections had to be filled with nitrogen, which was easily done by two or three times passing air through them and into the pyrogallol pipette and drawing it out again. On each subsequent occasion nothing but nitrogen from the last analysis was left in the connections, and the next analysis could be proceeded with at once. It did not, therefore, pay to make only one analysis a day, because in the course of the twenty-four hours diffusion through the rubber took place, but half a dozen samples could be analysed one after another at the rate of something like half an hour per sample for the three constituents. He agreed that sodium hyposulphite was quite satisfactory, but it did not last so long as the pyrogallol solution, one pipetteful of which would serve for 150 to 200 determinations. As to the combustion of methane, he had not been able to detect any trace that could not be burnt, and certainly as little as 0.01 per cent. of methane could with care be detected.

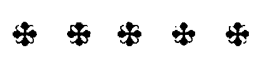

